\title{
An Inescapable Cat Odor Exposure Protocol for Studying Innate and Contextual Threat Conditioning in Rats
}

\author{
María Rodríguez $^{1,2}$, Marco Contreras ${ }^{3}$, Marcos Domic-Siede ${ }^{4}$, Francisco Ceric ${ }^{5}$, Fernando Torrealba ${ }^{1}$ \\ ${ }^{1}$ Departamento de Fisiología, Facultad de Ciencias Biológicas, Pontificia Universidad Católica de Chile ${ }^{2}$ Instituto de Ciencias Sociales, Universidad \\ de O'Higgins ${ }^{3}$ Department of Psychology, University of Arizona, Tucson ${ }^{4}$ Escuela de Psicología, Universidad Católica del Norte ${ }^{5}$ Laboratorio de \\ Neurociencia Afectiva, Facultad de Psicología, Universidad del Desarrollo
}

\section{Corresponding Author}

María Rodríguez

maria.rodriguez@uoh.cl

\section{Citation}

Rodríguez, M., Contreras, M., Domic-Siede, M., Ceric, F., Torrealba, F. An Inescapable Cat Odor Exposure Protocol for Studying Innate and Contextual Threat Conditioning in Rats. J. Vis. Exp. (177), e63078, doi:10.3791/63078 (2021).

DOI

$10.3791 / 63078$
URL

jove.com/video/63078

\section{Abstract}

Animals respond to threatening situations by exhibiting a number of defensive behaviors, including avoidance, freezing, and risk assessment. An animal model with an ethological approach offers a deeper insight into the biological mechanisms underlying threat responses. This paper describes a methodology to measure defensive behaviors toward both innate and learned aversive stimuli in rats. Animals were individually exposed to predator odor in an inescapable chamber to elicit a measurable, sustained, defensive state. The experimental design involved placing a rat in a familiar chamber for 10 min followed by exposure to cat odor for another $10 \mathrm{~min}$ in the same context. The next day, the rats were re-exposed for $10 \mathrm{~min}$ to the same context chamber where cat odor exposure occurred. Sessions were video-recorded and defensive behaviors were assessed on both days.

The behavioral test was coupled with reversible functional inactivation and c-Fos immunohistochemistry techniques to determine the role of the interoceptive cortex in threat responses. Rats exposed to cat odor on the first day and re-exposed to the context chamber on the second day displayed higher levels of defensive behaviors, and that cat odor elicited a robust increase in the neural activity of the interoceptive cortex. Moreover, muscimol inactivation of the interoceptive cortex reduced the expression of defensive behaviors in response to cat odor and impaired contextual threat memory. These results show that this behavioral assay is a useful tool for studying neural mechanisms of defensive behaviors and may offer insight into mechanisms that mediate fear in humans and its related disorders.

\section{Introduction}

Defensive behaviors occur in response to stimuli that signal a potential threat to an animal's survival. These behaviors are highly conserved across mammals and rapidly associated with stimuli or circumstances related to threat ${ }^{1,2,3}$. In nature, the threatening stimuli for most animals are predators; 
therefore, the detection of predator cues, such as odor cues, is particularly advantageous for avoiding predation. Behavioral responses to predator cues have been widely investigated in rodents.

For instance, stimuli, such as natural cat fur or skin odors, activate the olfactory and the vomeronasal system, inducing high levels of defensive behaviors ${ }^{4}$. These stimuli are accompanied by changes in neuronal and endocrine activity $^{5,6,7,8}$, and they are strong, unconditioned, aversive stimuli for contextual threat conditioning in rats $^{7,8,9,10,11}$. Studies have shown that at least $24 \mathrm{~h}$ after exposure to natural predator cues, rats display robust and long-lasting conditioned anxiety-like states ${ }^{7,12,13}$. This phenomenon is of particular interest for developing more realistic models of posttraumatic stress disorder (PTSD) $14,15,16,17$, generalized anxiety disorder $(G A D)^{5}$, and panic disorder $(\mathrm{PD})^{18,19}$.

In laboratory settings, fear behavior is measured as flight, avoidance (e.g., retreat, hiding), or freezing. Additionally, anxiety can be measured as stretch postures and vigilant scanning directed toward monitoring a predatory stimulusa set of responses broadly known as risk assessment behaviors $6,9,20$. Studies have shown that freezing behavior is the predominant defensive strategy in rats to an inescapable threat, whereas risk assessment is observed when the threat is ambiguous or non-localized ${ }^{12,21,32}$. Although it is known that an innate or learned stimulus can elicit defensive behaviors, there is a lack of laboratory behavioral paradigms that reliably capture defensive responses in a more ethological context. To fill this gap, we designed a protocol with an ethological approach that allows the measurement of sustained innate and contextual threat behaviors along with brain responses to naturalistic threat stimuli.

Stressful experiences, such as exposure to an inescapable predator odor, cause perdurable alterations in behavioral and physiological response in rats ${ }^{14,22,23}$. These alterations reflect the symptom profile observed in fear and anxietyrelated disorders such as PTSD. In the current model, a testing chamber is used without a safe hiding area to expose rats to an inescapable threat event and thus enhance defensive responses. Rats displayed robust freezing and risk assessment behaviors in response to both cat odor and the testing context. These findings support the use of this protocol as a reliable and valid method for exploring biological mechanisms underlying defensive behaviors and developing and refining new strategies for the treatment of fear disorders in humans.

\section{Protocol}

The following procedure was conducted according to the recommendations of the Institutional guidelines by the National Institutes of Health (USA) Guide for the Care and Use of Laboratory Animals (NIH Publication No. 80-23, revised 1996). The Institutional Bio-Safety and Ethical Committee at the Pontificia Universidad Católica de Chile approved all procedures. All experimental sessions were conducted during the active phase of the rat (dark phase).

\section{Preparation of the testing room and testing chamber}

NOTE: The overview of the apparatus is shown in Figure 1. The testing chamber was developed and modified according to previous studies 24,25 . 
1. Use a transparent rectangular poly (methyl methacrylate) testing chamber with the following dimensions: $60 \mathrm{~cm}$ (length) $\times 40 \mathrm{~cm}$ (width) $\times 40 \mathrm{~cm}$ (height). Cover the side and back walls of the testing chamber with any black paper. Ensure that the front of the testing chamber is transparent to record the animals' behaviors.

2. Fix a steel bracket to the floor at the bottom right corner of the testing chamber, and use it to attach the cat collars for the testing procedures.
3. Clean the testing chamber with $5 \%(\mathrm{v} / \mathrm{v})$ ethanol before and after the tests to remove any scent clues left by the subject rats and avoid the rat's aversion response. Allow 5 min between each session for the ethanol to evaporate fully.

4. Perform the testing session in a quiet room dimly lit by an $80 \mathrm{~W}$ red light bulb located $20 \mathrm{~cm}$ above the testing chamber.Use a video camera located in front of the chamber to record the animals' behavior.

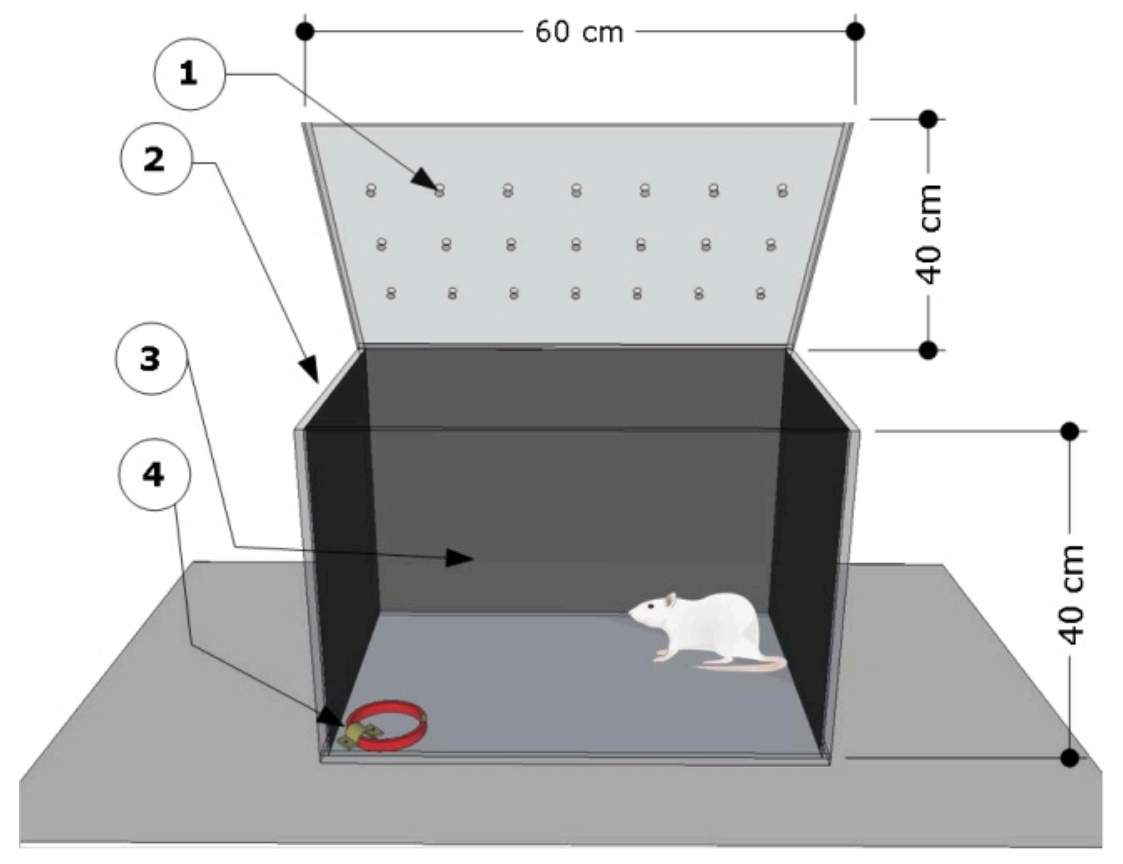

Figure 1: Schematic overview of the testing chamber. The apparatus consists of a test compartment with: (1) ventilation holes, (2) side, and (3) back walls darkened, and a steel bracket locked at the left corner floor (4) to attach the cat collar (dimensions: width $15 \mathrm{~mm}$, thickness $5 \mathrm{~mm}$, length $300 \mathrm{~mm})$. Chamber dimensions are indicated on the cartoon $(60 \mathrm{~L} \times 40 \mathrm{~W}$ $\mathrm{x} 40 \mathrm{H} \mathrm{cm}$ ). Please click here to view a larger version of this figure. 


\section{Cat odor preparation}

1. Obtain a cat collar with a felt-lined inner face to better trap the cat odor. Ensure the availability of several collars for use as control collar (no odor) or testing collar (cat odor). NOTE: The crucial point is that the cat collar must have an internal lining for collecting and trapping odors.

2. Put a collar on a domestic ovariectomized female cat and allow the cat to wear the collar for a week before testing. Try to keep the female cat indoors while wearing the testing collar.

NOTE: Use the same cat throughout the study.

3. Keep the worn cat collar in an airtight plastic container and store it at $4{ }^{\circ} \mathrm{C}$. Replace the worn cat collar with a collar containing a fresh cat odor every three days.

\section{Preparing rats for the experimental procedure}

1. Use adult male Sprague-Dawley rats that weigh $270-290$ g. House them in individual cages with chow and water available ad libitum.

2. [Optional] Preparing rats for surgical procedure (optional)

1. Perform the surgery under sterile conditions. Clean and disinfect the working area with a solution of $70 \%$ ethanol and sterilize all surgical materials and instruments.

2. Anesthetize the rat with an intraperitoneal mixture of ketamine $(100 \mathrm{mg} / \mathrm{kg})$ and xylazine $(20 \mathrm{mg} /$ $\mathrm{kg})$. Wait until the proper plane of anesthesia is achieved when the rat does not respond to a toe pinch stimulus or corneal stimulation. To maintain the anesthetic condition throughout the surgery, administer an additional dose of the anesthetic cocktail every $45 \mathrm{~min}$.
3. Gently shave the rat's head from behind the ear to between the eyes, and disinfect the surgical area with a sterile alcohol preparation pad followed by a povidone-iodine solution. Apply lubricant eye ointment to avoid corneal drying during the surgical procedure.

4. Place the rat in a stereotaxic apparatus, and hold the head by properly positioning the ear bars in the ear canals. Then, gently insert the rat's incisors in the incisor bar and secure the nose clamp.

5. Place a sterile $26 \mathrm{G}$ stainless-steel guide cannula in the stereotaxic holder. Take a scalpel and make a midline incision along the scalp. Place 2 or more clamps around the incision $(2-3 \mathrm{~cm})$.

6. Clear the skull completely of tissue until both bregma and lambda cranial sutures are visible.

7. Verify the symmetrical positioning of the head by the alignment of the dorsoventral and anteroposterior axis of the skull using the incisor and ear bars, respectively. Identify the coordinates of the guide cannula for the brain region of interest. Keep the bregma and lambda in the same horizontal plane using the incisor bar.

8. Calculate the coordinates of the guide cannula implantation site into the area of interest following the stereotaxic coordinates of Paxinos and Watson $^{26}$ or Swanson atlas ${ }^{27}$.

NOTE: For the primary interoceptive insular cortex, the following stereotaxic coordinates were used: Bregma $-0.51 \mathrm{~mm}$, midline $5.0 \mathrm{~mm}$, depth from the cranial surface $4.5 \mathrm{~mm}$, angled $10^{\circ}$ medially from vertical. 
9. Check the guide cannula implantation coordinates by bringing it down until it touches the skull, and then drill a hole through the cranium on the verified site. Drill 3 additional holes around the target area to place 3 screws into the skull.

10. Insert the cannula into the brain area of interest and use screws and dental acrylic to anchor the cannula to the skull. Pour dental acrylic on the dry skull and ensure that it covers the screws to fix the cannula in place. Allow the acrylic dental to harden and then remove the stereotaxic arm carefully, leaving the guide cannula in place.

NOTE: This procedure immobilizes the implanted cannula and allows experimenters to perform multiple microinjections in the area of interest.

11. Insert a stainless-steel stylet into the guide cannula to avoid obstruction and contamination.

12. At the end of the surgery, administer a single dose of antibiotic (enrofloxacin 5\%; $19 \mathrm{mg} / \mathrm{kg}$ i.p) and antiinflammatory (ketophen $0.2 \mathrm{mg} / \mathrm{kg}$ i.p.). Repeat this step for three consecutive days after the surgery.

13. Allow the rats to fully recover for at least one week before starting the experiments.

3. Keep the rats under a reversed $12 / 12 \mathrm{~h}$ light/dark cycle (lights on at 7:00 P.M.) for at least ten days before the start of the experiments (Figure 2).
NOTE: If the experimenter needs to perform any surgical procedure, it would be necessary to do it before reversing the dark-light cycle. This allows for the reduction of stress after surgery.

4. Handle all rats for $20 \mathrm{~min} /$ day for $2-3$ days. Take one rat at a time, hold the animal securely by the torso (not by the tail), and pet them during this time. If animals show signs of stress, handle them for extended periods.

NOTE: This step is critical to minimize handling stress that may affect the following steps.

5. Preexpose the animals to the testing chamber (cat odor chamber with an unworn cat collar) for $30 \mathrm{~min} /$ day for three days before the experiments to allow the animals to acclimate to the testing context.

NOTE: It is suggested that the experimenter should not wear scented perfumes or lotions.

6. After placing the animal in the chamber, wait for approximately 10-15 min, introduce an arm into the testing chamber, and swap the cat collar for another cat collar with the same features.

NOTE: This step is critical to simulate, as closely as possible, each step of the behavioral procedures to minimize stress, which may affect the behavioral performance of the rat in the last 10 min of the test.

7. Clean the testing chamber with $5 \%(\mathrm{v} / \mathrm{v})$ ethanol between sessions. 


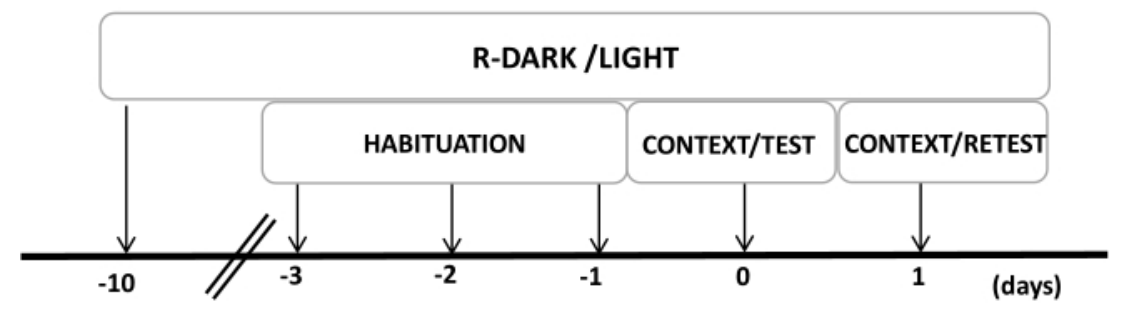

Figure 2: Timeline of the experimental design. Rats were exposed to a reversed dark-light cycle for 10 days and then habituated to the testing chamber containing a control (unworn) cat collar for $30 \mathrm{~min}$ for the last three days. On day 0 , rats were first exposed to a familiar testing chamber (CONTEXT) for $10 \mathrm{~min}$ and then exposed to a collar with or without cat odor for an additional period of $10 \mathrm{~min}$ (TEST) in the same context. On day 1, rats exposed to cat odor (TEST) on day 0 were returned to the same testing chamber for $10 \mathrm{~min}$ (CONTEXT) and re-exposed to cat odor (RETEST) for an additional period of $10 \mathrm{~min}$. This figure has been modified from ${ }^{8}$. Abbreviation: R-Dark/light = reverse dark-light cycle. Please click here to view a larger version of this figure.

\section{Cat odor test procedures}

1. On Day 0 of the experimental protocol, assess threat responses to the cat odor.

2. If necessary, administer cortical injections before the behavioral tests following the method described below. If performing a behavioral protocol only, skip this step and proceed to step 4.3.

1. Insert a sterile $33 \mathrm{G}$ injection cannula coupled to a $1 \mu \mathrm{L}$ Hamilton syringe into the guide cannula after removing the occluder.

2. Fill the Hamilton syringe with sterile saline (control rats) or the GABA-A receptor agonist, muscimol (treatment rats), and inject $0.5 \mu \mathrm{L} /$ hemisphere over
$2 \mathrm{~min}$. Wait for $2 \mathrm{~min}$ to allow diffusion of the drug, slowly remove the injection cannula, and immediately insert the occluders.

NOTE: Perform this step in a quiet, awake animal and inject the drugs slowly to minimize jitter of the injection cannula and consequent diffusion to adjacent brain areas.

3. Once the cortical injection/hemisphere is completed, wait for $30 \mathrm{~min}$ and proceed to the next step.

3. Place the animals in the testing chamber for $20 \mathrm{~min}$. During the first $10 \mathrm{~min}$, expose the animals to an unworn familiar collar. Introduce the collar impregnated with cat odor (TEST) or an unworn familiar collar (CONTEXT) by the next 10 min (Figure 3). 

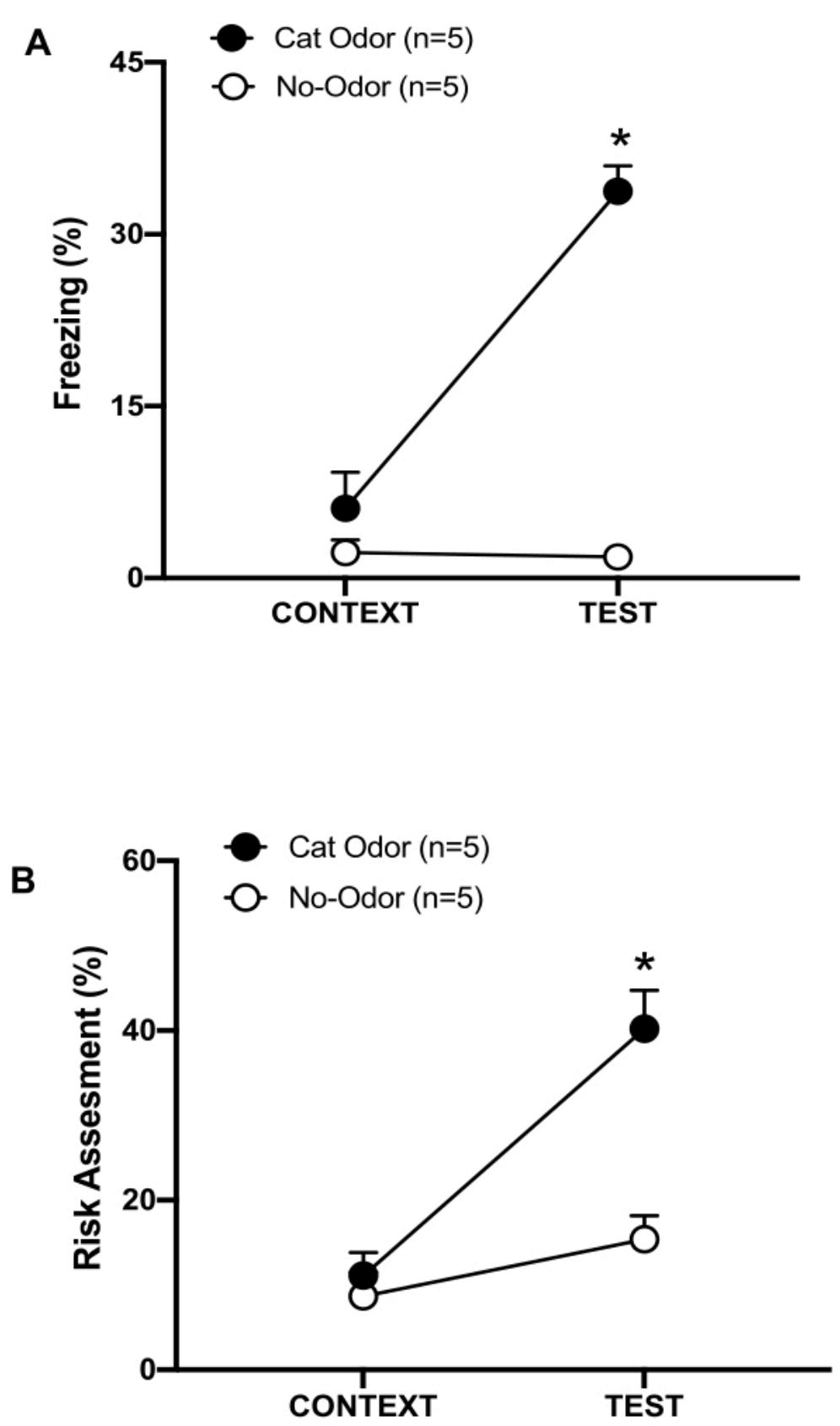

Figure 3: Cat odor encounter increases innate defensive behaviors. (A, B) Rats were first exposed to the familiar testing chamber (CONTEXT) for 10 min and then exposed to a collar with cat odor (cat odor group, black circles) or without cat odor (no-odor group, open circles) for an additional period of $10 \mathrm{~min}$ (TEST) in the same context. The circles show the percentage of time spent in freezing $(\mathbf{B})$ and risk assessment $(\mathbf{C})$ displayed by naïve rats. Data are expressed as means + SEM. ${ }^{*} p<$ 0.05 . This figure has been modified from ${ }^{8}$. Please click here to view a larger version of this figure. 
4. Place a video camera in front of the testing chamber and record the animal's behavior for later behavioral scoring by an experimenter blind to the experimental conditions.

5. At the end of the cat odor session, return the animals to their home cages and put them back in the animal facility until the next session.

6. Clean the testing chamber with $5 \%(\mathrm{v} / \mathrm{v})$ ethanol between sessions.

7. Twenty-four hours after the cat odor session (Day 1), assess defensive responses to the testing context.

8. If needed, repeat step 4.2 before context exposure.
9. Return the rats to the same testing chamber with the unworn familiar collar, and score for defensive behaviors to measure contextual threat memory during $10 \mathrm{~min}$ (Figure 4).

10. Place a video camera in front of the testing chamber and record the animal's behavior for later behavioral scoring by an experimenter blind to the experimental conditions.

11. During the next $10 \mathrm{~min}$, replace the unworn familiar collar, re-expose the animal to a collar with cat odor, and measure defensive behaviors.

12. After the second exposure to cat odor, return the animal to its home cage, and clean the testing chamber with $5 \%$ $(v / v)$ ethanol. Put the rat back in the animal facility.

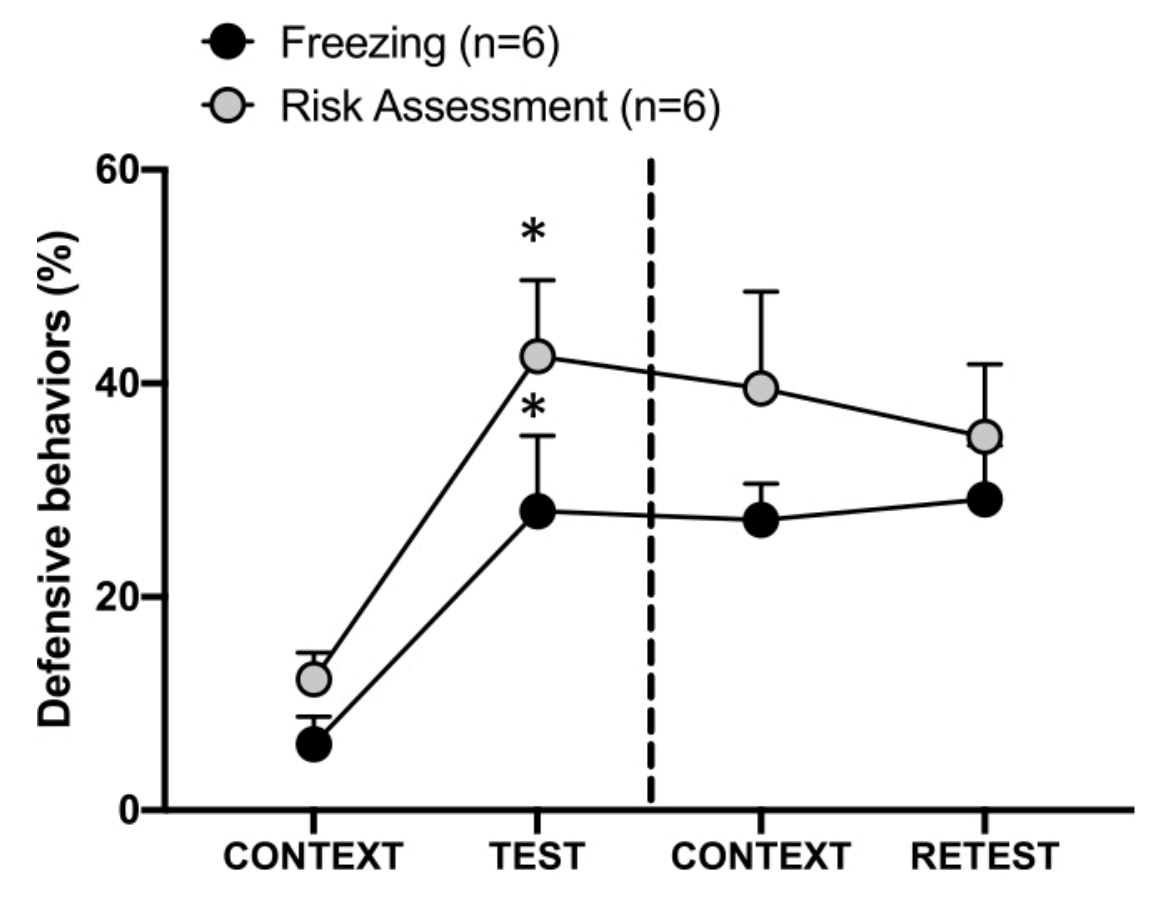

Figure 4: Cat odor encounter induces contextual threat learning. The circles show the percentage of time spent in freezing (black circles) and risk assessment behavior (gray circles). The dashed line separates the innate fear test (Day 0 , left) from the contextual fear test (Day 1, right). Data are expressed as means + SEM. ${ }^{*} p<0.05$. This figure has been modified from ${ }^{8}$. Please click here to view a larger version of this figure. 


\section{Immunohistochemical procedures}

1. Anesthetize the animal with an intraperitoneal mixture of ketamine $(0.45 \mathrm{mg} / \mathrm{kg})$ and xylazine $(0.05 \mathrm{mg} / \mathrm{kg}) 90 \mathrm{~min}$ after completing the experiments. Then, euthanize the rat by transcardial perfusion with $4 \%$ paraformaldehyde.

NOTE: If it is necessary to evaluate the first cat odor exposure, conditioned response to the context, or reexposure to cat odor, perform this step after each of these experimental situations. Wear gloves and lab coat and handle paraformaldehyde with care under a chemical fume hood.

2. After perfusion, fix the brain in the same fixative solution for $2 \mathrm{~h}$ and then transfer it to $30 \%$ sucrose with $0.02 \%$ sodium azide in phosphate-buffered saline (PBS) until the brain sinks to the bottom. Store the brain at $4{ }^{\circ} \mathrm{C}$.

3. Freeze the brain using dry ice and cut it into $50-\mu m$-thick serial coronal sections on a sliding microtome. Collect three sets of brain sections of the area of interest, leaving one for verification of cannula placement and two sets for the immunohistochemical procedure.

4. Incubate the free-floating brain sections in $0.3 \% \mathrm{H}_{2} \mathrm{O}_{2}$ in PBS for 30 min, wash in PBS, and transfer these sections to the blocking $(0.4 \%$ Triton $X-100,0.02 \%$ sodium azide, $3 \%$ normal goat serum in PBS) solution for $1 \mathrm{~h}$.

5. Transfer the sections to the primary antibody incubation solution containing c-Fos antibody diluted 1:20,000 in blocking solution and leave them overnight at room temperature.
6. Rinse the sections in PBS for $1 \mathrm{~h}$ and then incubate them in the secondary antibody solution diluted 1:1,000 in $0.4 \%$ Triton $\mathrm{X}-100$ and $1.5 \%$ normal goat serum in PBS.

7. Rinse the sections for $40 \mathrm{~min}$ and incubate them for $\mathbf{1}$ $\mathrm{h}$ in the avidin/biotin-based peroxidase system (see the Table of Materials), diluted 1:500 in PBS.

8. Rinse and incubate the sections in a $0.05 \%$ solution of 3-3' diaminobenzidine hydrochloride (DAB) containing $0.003 \% \mathrm{H}_{2} \mathrm{O}_{2}$ and $0.05 \%$ nickel chloride to obtain a dark blue reaction product.

\section{Cell counting}

1. Examine sections under a camera lucida using a low power (10x) objective to localize the c-Fos punctiform staining in the nuclei of neurons (Figure 5).

2. Use a counting grid related to the size of the area of interest. For the pIC, for example, from Bregma 0.95 to -0.26 , use a $0.25 \mathrm{~mm} \times 1 \mathrm{~mm}$ counting grid; from Bregma -0.51 to $-2.45 \mathrm{~mm}$, use a $0.5 \mathrm{~mm} \times 1 \mathrm{~mm}$ counting grid.

3. Quantify the number of c-Fos immunoreactive (Fos-ir) cells per section using a digit manual counter.

4. Count all coronal sections of interest twice and ensure that the same observer, blind to the experimental conditions, performs the counting.

5. Take a photograph using a microscope coupled with a digital camera. 
A
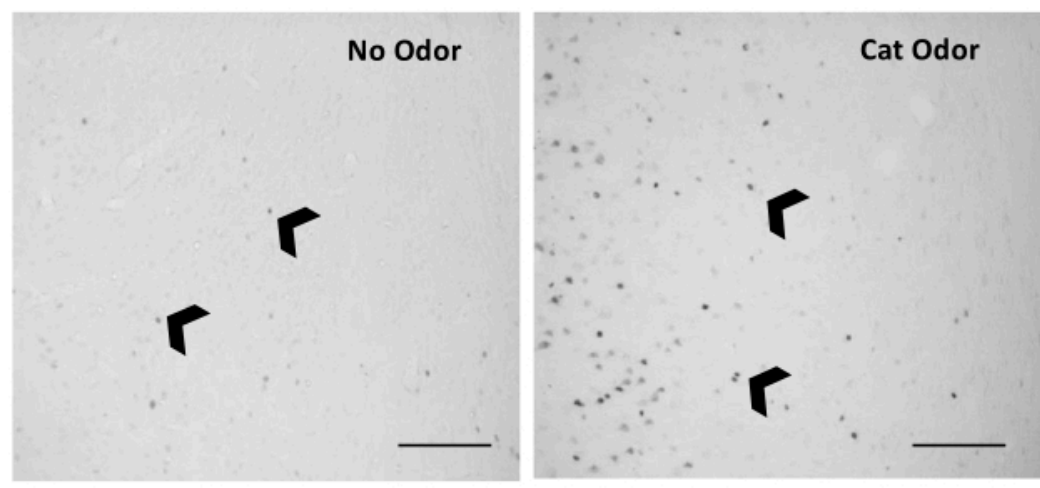

B

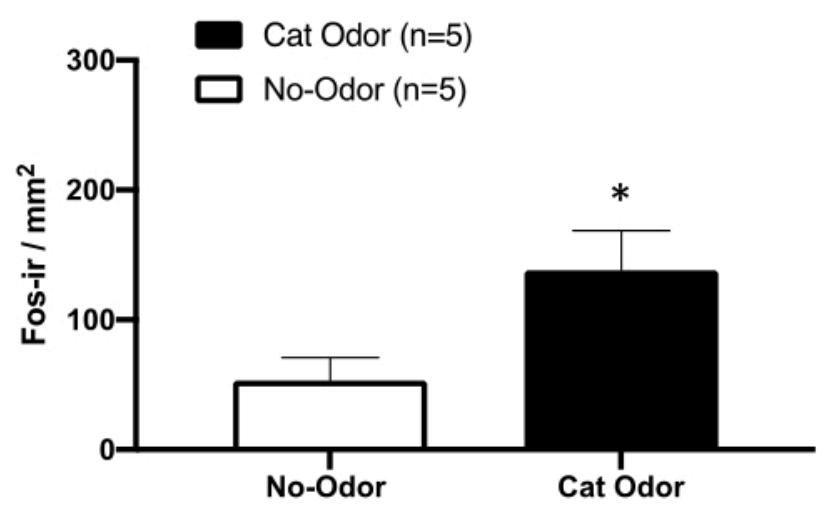

Figure 5: Cat odor elicits neuronal activation in the primary interoceptive cortex. (A) Representative photomicrograph of the pIC showing a near absence of c-Fos-ir cells (black arrowheads) in rats exposed to an unworn cat collar (no odor, left) compared with a remarkably increased number of c-Fos-ir cells in rats exposed to cat odor (right). (B) Quantification of cFos-ir cells in the pIC in both experimental conditions. Data are expressed as means + SEM. ${ }^{*} p<0.05$. Scale bars $=200$ $\mu \mathrm{m}$. This figure has been modified from ${ }^{8}$. Abbreviations: $\mathrm{pIC}=$ primary interoceptive cortex; $\mathrm{c}$-fos-ir $=\mathrm{c}$-Fos-immunoreactive. Please click here to view a larger version of this figure.

\section{Data analysis}

1. Score defensive behaviors from the video-recorded experiments and analyze them using statistical software.

2. Evaluate freezing by timing bouts, expressed as a percentage of the time spent freezing during $10 \mathrm{~min}$. Calculate the percentage of freezing using Eq (1).

Freezing $=($ seconds spent freezing $/ 600 \mathrm{~s}) \times 100(\mathbf{1})$
NOTE: Freezing was defined as the complete lack of movement except for respiration for at least $1 \mathrm{~s}^{28}$.

3. Score risk assessment by timing vigilant scanning bouts and express it as a percentage of the time spent in risk assessment during $10 \mathrm{~min}$. Calculate the percentage of risk assessment using Eq (2).

$\%$ Risk assessment $=$ (seconds spent in risk assessment/600 s) $\times 100(2)$ 
NOTE: Risk assessment behavior was defined as vigilant scanning (observatory, side-to-side head movements, without locomoting) for at least $1 \mathrm{~s}^{29}$.

4. Score these behavioral parameters manually and ensure that they are counted by an experimenter blinded to the experimental conditions (i.e., odor and drug conditions). Depending on the normality of the data sets, use parametric or nonparametric tests for comparing two or more groups.

NOTE: In this study, all statistical tests were performed using SPSS software.

1. Use the Kruskal-Wallis $H$-test with the MannWhitney $U$-test for pairwise comparisons between groups.

2. Analyze intragroup comparisons over time with the Friedman test followed by the Wilcoxon signed-rank test for pairwise comparisons.

NOTE: In all figures, significance levels were set to $\mathrm{p}<0.05\left(^{*}\right)$ and $\left.\mathrm{p}<0.01{ }^{* *}\right)$.

5. For the analysis of c-Fos expression in specific brain areas, count the number of c-Fos-positive cells per section by calculating the density of c-Fos-ir cells per $\mathrm{mm}^{2}$, and compare the mean number between control (no odor) and stimulated (cat odor) conditions.

1. Depending on the normality of the data, use unpaired student's $t$-test or Mann-Whitney $U$-test.

NOTE: In this study, the Mann-Whitney U-test was used; differences were considered significant if $p<$ $0.05\left(^{*}\right)$.

\section{Representative Results}

In this protocol, the freezing and risk assessment percentages were measured as indicators of fear and anxiety-like states in rats, respectively. The timeline of the experimental design is shown in Figure 2. The results from the animals exposed to cat odor on day 0 are presented in Figure 3. Rats showed significantly higher levels of freezing (Figure 2A, Cat Odor group, Wilcoxon Signed-ranks test, $Z=-2.201, p=0.028$ ) and risk assessment (Figure 2B, Cat Odor group, Wilcoxon Signed-ranks test $Z=-2.336 p=0.018$ ) in response to cat odor (TEST) than to familiar context (CONTEXT). Low levels of freezing (Figure 2A, no-odor group, Wilcoxon Signedranks test, $Z=-0.184, p=0.854$ ) and risk assessment (Figure 2B, no-odor group, Wilcoxon Signed-ranks test, $Z=-1.753$, $p=0.08$ ) were observed in rats exposed to an unworn collar during the second part of the test.

Additional analysis revealed that the levels of freezing (Figure 2A, Mann-Whitney test, $U=0.000, p=0.004$ ) and risk assessment (Figure 2B, Mann-Whitney test, $U=4.000, p$ $=0.025)$ were higher in the Cat Odor group than the noodor group during the second part of the test. There were no significant differences in freezing (Figure 2A, Mann-Whitney test, $U=11.000, p=0.256$ ) and risk assessment (Figure 2B, Mann-Whitney test, $U=15.00, p=0.627$ ) between the two groups during the first $10 \mathrm{~min}$ of exposure to a familiar context (CONTEXT). These results demonstrate that the protocol is suitable for testing defensive behaviors in response to cat fur/ skin odor.

The conditioned threat response to the testing context (CONTEXT) on day 1 is shown in Figure 4. Rats exposed to cat odor were returned to the testing chamber $24 \mathrm{~h}$ after the first predator's odor encounter. On day 1 , animals exhibited higher freezing (Wilcoxon Signed-ranks test, $Z=-2.366, p$ $=0.018$ ) and risk assessment (Wilcoxon Signed-ranks test, $Z=-2.201, p=0.028$ ) levels in response to the testing context than on day 0 . In addition, there were no differences in 
freezing (Wilcoxon Signed-ranks test, $Z=-0.841, p=0.400$ )

or risk assessment (Wilcoxon Signed-ranks test, $Z=-0.943$, $p=0.345)$ levels between CONTEXT and RETEST. The rats showed the same freezing levels (Wilcoxon Signed-ranks test, $Z=-0.105, p=0.917$ ) and risk assessment (Wilcoxon Signed-ranks test, $Z=-0.980, p=0.327$ ) during RETEST and TEST. These results demonstrated that a single $10 \mathrm{~min}$ cat fur/skin odor exposure resulted in a learned threat response to the context in which the animals were confronted with predator odor.

Figure 5 and Figure 6 show two sets of experiments using the protocol presented in this article. We tested whether the primary interoceptive cortex $(\mathrm{plC})$, a brain region involved in emotion processing $8,30,31,32$, is necessary for the expression of defensive behaviors. Cat odor-induced neuronal activation was assessed in the pIC by counting c-Fos-ir cells in separated animal groups: no-odor and cat odor conditions. These rats were euthanized $90 \mathrm{~min}$ after the completion of the TEST. A significant increase was observed in the number of c-Fos-ir neurons in the pIC in the cat odor group (Mann-Whitney test, $U=3.000, p=0.016$ ) compared with the control group (Figure 5). 


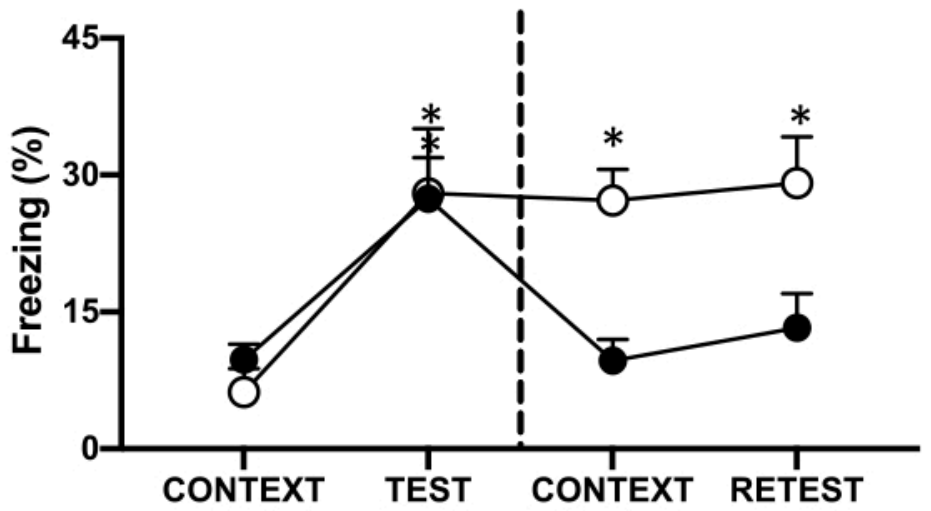

B

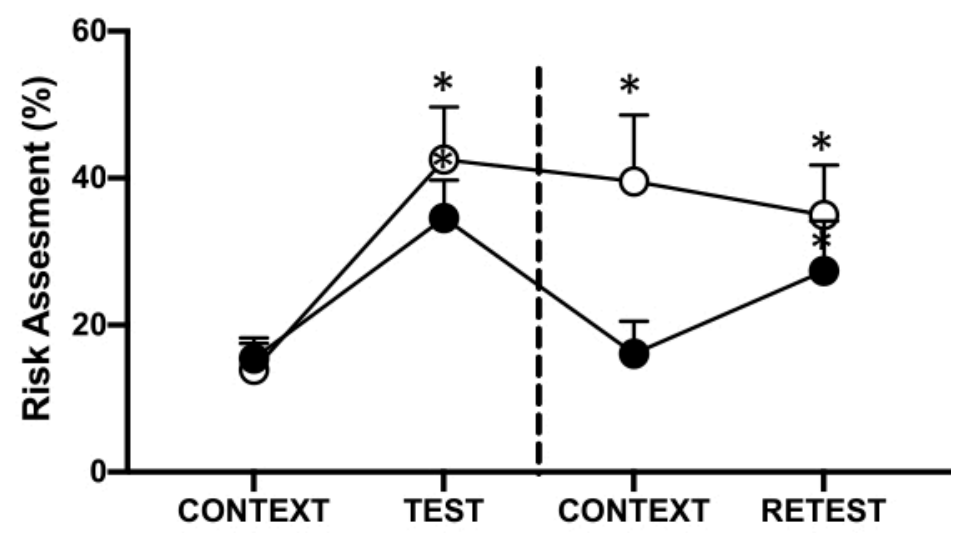

Figure 6: Muscimol inactivation of the primary interoceptive cortex impairs contextual threat memory. (A, B)

Treatment group was injected with saline on day 0 and muscimol on day 1 in the pIC (Treatment group). Control rats were injected with saline in the pIC on both days. On day 1 , animals were returned to the familiar testing chamber and exposed to the CONTEXT for $10 \mathrm{~min}$ and re-exposed to cat odor (RETEST) for an additional period of $10 \mathrm{~min}$. The circles show the percentage of time spent in freezing (black circles) and risk assessment behavior (open circles). The dashed line separates the innate fear test (Day 0, left) from the contextual fear test (Day 1, right). Data are expressed as means + SEM. ${ }^{*} p<0.05$. This figure has been modified from ${ }^{8}$. Abbreviations: $\mathrm{pIC}=$ primary interoceptive cortex; Sal-Sal $=$ saline injected on day 
0 and day 1 ; Sal-Mus = saline injected on day 0 and muscimol on day 1. Please click here to view a larger version of this figure.

We also measured the effect of muscimol inactivation of the pIC on contextual threat memory (Figure 6). The treatment group (sal-mus rats) received a saline injection in the $\mathrm{pIC}$ on day 0 and GABA-A agonist muscimol on day 1 . This group of animals did not show any differences in freezing (Figure 6A, Wilcoxon Signed-ranks test, $Z=-0.140, p=0.889$ ) or risk assessment (Figure 6B, Wilcoxon Signed-ranks test, Z $=-0.700, p=0.484$ ) levels on day 1 in response to the familiar context compared to day 0 , indicating an impairment in contextual threat memory. Interestingly, freezing (Figure 6A, Wilcoxon Signed-ranks test, $Z=-2.100, p=0.036$ ), but not risk assessment (Figure 6B, Wilcoxon Signed-ranks test, $Z=-0.980, p=0.327$ ), was significantly reduced during RETEST compared to TEST. This last finding indicates that silencing the pIC on day 1 selectively impaired the fear, but not anxiety, response to the predator scent. Taken together, these results support that the experimental protocol described above is suitable for the study of innate and learned defensive responses to predatory threats.

\section{Discussion}

The protocol described here offers an innovative approach to assess defensive behaviors evoked by innate and learned aversive stimuli. A testing chamber without a safe hiding area (Figure 1) and a collar impregnated with skin/fur odor from an ovariectomized female domestic cat were used to elicit a strong and sustained threat state in rats that may be useful to investigate neural circuits underlying adaptive and maladaptive defensive reactions.

It is well known that the display of specific defensive reactions depends on the features of both the threat stimulus and the situation/environment in which the animal is encountered $^{21,33}$. Avoidance, risk assessment, and freezing are part of a vast repertoire of animal defensive reactions that can be evoked by threatening stimuli ${ }^{9,19}$. However, the selection of the predominant defensive reaction depends on the environmental conditions such as the distance from the threat ${ }^{33}$ or the presence of a safe place inside the testing chamber 21,33 . For instance, when rats are placed in the arena with a hiding box, in the presence of cat odor, they display defensive behaviors such as stretch/attend postures, head-out-of-the-hide box, and avoidance $6,24,25$. In contrast, freezing behavior, vigilant scanning, and stretch/ attend postures are strongly elicited in situations when escape is not possible, and the distance to the threat is not overly short ${ }^{33,34}$.

Studies have shown that exposure to an uncontrollable stressor produces a variety of behavioral consequences that are different from those that occur when the stressor is controllable $35,36,37,38$. For example, inescapable, but not escapable, tail shock leads to large increases in serotonin in the dorsal raphe nucleus ${ }^{35}$ and anxiety-like behaviors measured $24 \mathrm{~h}$ after the aversive experience ${ }^{36}$. Moreover, uncontrollable stressors enhance fear conditioning in animals ${ }^{36,37}$ and humans ${ }^{38}$. Our initial rationale for developing the protocol was to expose rats to a situation where they cannot control the aversive stimulus, and therefore display strong and sustained threat responses and develop enhanced contextual learning after a single and short cat odor exposure.

In the experimental design described here, the absence of a hiding box evoked a strong and sustained defensive state that 
alternated between freezing (i.e., complete immobility except breathing ${ }^{28}$ ) and risk assessment (i.e., vigilant scanning and stretch/attend postures ${ }^{29}$ ) behaviors, which are usually considered as behavioral manifestations of fear and anxietylike states in rodents, respectively (Figure 3). Notably, the same pattern of defensive reactions occurred $24 \mathrm{~h}$ later when the rats were re-exposed to the testing context, indicating that a single $10 \mathrm{~min}$ cat fur/skin odor exposure is sufficient to induce long-lasting contextual threat learning, as previously reported $^{7,10,11,14,15,34,39}$ (Figure 4).

A cat collar with an internal felt lining was used for collecting and trapping odors/scents efficiently and thus obtain a reliable sample of the threat stimulus to evoke a strong defensive response. Researchers have used aversive stimuli such as cat feces, urine, or trimethylthiazoline (TMT, a component of fox feces) in similar work. Nevertheless, these stimuli seem to be less predictive of the immediate presence of a predator because they are less capable of inducing contextual learning 40,41 . According to previous findings $2,4,8,9,10,11,20,34,39$, cat odor is a reliable innate aversive stimulus that can induce sustained defensive reactions and contextual threat memory in rats. Over the years, this kind of ethological behavioral animal model has increasingly captured the interest of researchers to study stress and stress-related disorders ${ }^{13,14,15,16,17,23,42}$ like those associated with maladaptive fear memories such as PTSD.

This protocol is intended to be used in conjunction with a variety of experimental techniques, including, for example, molecular and cell biology approaches and electrophysiology in awake and behaving animals, which offer the opportunity to answer open questions and improve our understanding of adaptive and maladaptive threat responses. In this study, we tested the idea that the plC, a brain region involved in emotion processing, is necessary for the expression of defensive behaviors. Behavioral experiments were coupled with c-Fos immunohistochemistry to map patterns of neuronal activity in the pIC in response to cat odor and intracerebral infusions of the GABA-A receptor agonist, muscimol, to reversibly silence the $\mathrm{pIC}$ and determine its involvement in innate and learned threat reactions to predator odor. These findings revealed that cat odor elicited an increase in neuronal activity in the pIC (Figure 5), and that silencing the pIC led to a severe deficit of contextual threat memory (Figure 6).

Although the protocol described here is technically simple to implement and perform, a few complications may arise. For example, cat odor cross-contamination may occur if the unworn collar comes in contact with the worn cat collar. Thus, the collars must be kept separately during all procedures, and gloves should be changed after placement of the worn collar in the testing chamber. If the experimenter wants to conduct odor and no-odor conditions using the same testing chamber, the experiments must be performed on different days. Two identical testing chambers could be used for these experiments $^{34}$, and the rats should be maintained in separate rooms to avoid social communication ${ }^{43}$. The stress elicited by exposure to novel stimuli in unfamiliar environments may also be an issue. Hence, the animals must have at least three days of habituation to the testing environment and procedures to reduce the stress and defensiveness commonly displayed by rats in novel situations. In addition, the time required for the habituation period should be longer than the test period. For instance, if the test takes $10 \mathrm{~min}, 20$ or $30 \mathrm{~min}$ should be assigned for the habituation period.

Finally, defensive behaviors should be preferably evaluated during the dark phase of the cycle, when rats are active. The 
rats must be maintained under a reverse light/dark cycle to allow the experimental procedures to be performed while both the rat and the experimenter are in their active phases ${ }^{34}$. The change in the light/dark cycle takes no more than 10 days to be settled, and based on experience, most rats under this condition respond adequately to the cat odor. However, there are a few studies that show that the Sprague-Dawley rats are less vulnerable to long-lasting threat conditioning and anxiety than Wistar rats ${ }^{44}$. Thus, it is possible that the use of Wistar rats instead of Sprague-Dawley strain rats could yield more robust results.

In conclusion, cat odor is an ethologically relevant threatening stimulus that elicits reliable neuronal, endocrine, and behavioral responses in rats. The encounter with cat odor in an inescapable chamber leads to a robust and prolonged fear/anxiety response in rats, which results in rapid and durable contextual threat learning. The protocol described above could be a useful tool for studying fear and adaptive and maladaptive threat memory encoding mechanisms.

\section{Disclosures}

The authors of this manuscript have no competing financial interests or other conflicts of interest to disclose.

\section{Acknowledgments}

The authors gratefully acknowledge Marcela Gonzalez for helping with laboratory procedures, Mabel Matamala for contributing to the protocol design, and Miguel Rojas for helping with illustration.

\section{References}

1. Steimer, T. The biology of fear- and anxiety-related behaviors. Dialogues in Clinical Neuroscience., 4 (3), 231-249 (2002).

2. Gross, C. T., Canteras, N. S. The many paths to fear. Nature Reviews. Neuroscience. 13 (9), 651-658 (2012).

3. Harrison, L. A., Ahn, C., Adolphs, R. Exploring the structure of human defensive responses from judgments of threat scenarios. PLoS One., 10 (8), e0133682 (2015).

4. Papes, F., Logan, D. W., Stowers, L. The vomeronasal organ mediates interspecies defensive behaviors through detection of protein pheromone homologs. Cell. 141 (4), 692-703 (2010).

5. Blanchard, R. J., Griebel, G., Andrew Henrie, J., Blanchard, D. C. Differentiation of anxiolytic and panicolytic drugs by effects on rat and mouse defense test batteries. Neuroscience and Biobehavioral Reviews. 21 (6), 783-789 (1997).

6. Dielenberg, R. A., McGregor, I. S. Defensive behavior in rats towards predatory odors: a review. Neuroscience and Biobehavioral Reviews. 25 (7-8), 597-609 (2001).

7. Muñoz-Abellan, C., Daviu, N., Rabasa, C., Nadal, R., Armario, A. Cat odor causes long-lasting contextual fear conditioning and increased pituitary-adrenal activation, without modifying anxiety. Hormones and Behavior. 56 (4), 465-471 (2009).

8. Rodriguez, M. et al. Interoceptive insular cortex mediates both innate fear and contextual threat conditioning to predator odor. Frontiers in Behavioral Neuroscience. 13, $283(2020)$. 
9. Blanchard, R. J., Yang, M., Li, C. I., Gervacio, A., Blanchard, D. C. Cue and context conditioning of defensive behaviors to cat odor stimuli. Neuroscience and Biobehavioral Reviews. 25 (7-8), 587-595 (2001).

10. Takahashi, L. K., Chan, M. M., Pilar, M. L. Predator odor fear conditioning: current perspectives and new directions. Neuroscience and Biobehavioral Reviews. 32 (7),1218-1227 (2008).

11. de Lima, M. A. X., Baldo, M. V. C., Canteras, N. S. Revealing a cortical circuit responsive to predatory threats and mediating contextual fear memory. Cerebral Cortex. 29 (7), 3074-3090 (2019).

12. Blanchard, D. C., Blanchard, R. J. Defensive behaviors, fear and anxiety. In Handbook of anxiety and fear. Blanchard, R. J., Blanchard, D. C., Griebel, G., Nutt, D. J. (Eds), Elsevier Academic Press, Amsterdam, 63-76 (2008).

13. Armario, A., Escorihuela, R. M., Nadal, R. Long-term neuroendocrine and behavioural effects of a single exposure to stress in adult animals. Neuroscience and Biobehavioral Reviews. 32 (6), 1121-1135 (2008).

14. Cohen, H., Matar, M. A., Joseph, Z. Animal models of posttraumatic stress. Preclinical models of neurologic and psychiatric disorders. Current Protocols in Neuroscience. Chapter 9, Unit 9, 45 (2013).

15. Liang, Z., King, J., Zhang, N. Neuroplasticity to a singleepisode traumatic stress revealed by resting-state fMRI in awake rats. Neurolmage. 103, 485-491(2014).

16. Dengler, B. A., Hawksworth, S. A., Berardo, L., McDougall, I., Papanastassiou, A. M. Bilateral amygdala stimulation reduces avoidance behavior in a predator scent post-traumatic stress disorder model. Neurosurgical Focus. 45 (2), E16 (2018).

17. Ozbeyli, D. et al. Protective effects of vortioxetine in predator scent stress model of post-traumatic stress disorder in rats: role on neuroplasticity and apoptosis. Journal of Physiology and Pharmacology. 70 (4), 557-571 (2019).

18. Graeff, F. G., Del-Ben C. M. Neurobiology of panic disorder: From animal models to brain neuroimaging. Neuroscience and Biobehavioral Reviews. 32 (7), 1326-1335 (2008).

19. Blanchard, D. C., Griebelb, G., Pobbec, R., Blanchard, R. J. Risk assessment as an evolved threat detection and analysis process. Brain Research. 35 (4), 991-998 (2011).

20. Apfelbach, R., Blanchard, C. D., Blanchard, R. J., Hayes, R. A., McGregor, I. S. The effects of predator odors in mammalian prey species: a review of field and laboratory studies. Neuroscience and Biobehavioral Reviews. 29 (8), 1123-1144 (2005).

21. Blanchard, D. C., Blanchard, R. J., Griebel, G. Defensive responses to predator threat in the rat and mouse. Current Protocols in Neuroscience. Chapter 8, Unit 8.19 (2005).

22. Zoladz, P. R., Conrad, C. D., Fleshner, M., Diamond, D. M. Acute episodes of predator exposure in conjunction with chronic social instability as an animal model of posttraumatic stress disorder. Stress. 11 (4), 259-281 (2008).

23. Shallcross, J. et al. The divergent effects of CDPPB and cannabidiol on fear extinction and anxiety in a predator scent stress model of PTSD in rats. Frontiers in Behavioral Neuroscience. 13, 91 (2019). 
24. Dielenberg, R. A., Carrive, P., McGregor, I. S. The cardiovascular and behavioral response to cat odor in rats: unconditioned and conditioned effects. Brain Research. 897 (1), 228-237 (2001).

25. Dielenberg, R. A., Leman, S., Carrive, P. Effect of dorsal periaqueductal gray lesions on cardiovascular and behavioral responses to cat odor exposure in rats. Behavioral Brain Research. 153 (2), 487-496 (2004).

26. Paxinos, G., Watson, C. The rat brain in stereotaxic coordinates. San Diego: Academic Press. (1997).

27. Swanson, L. W. Brain maps: Structure of the rat brain. A laboratory guide with printed and electronic templates for data, models, and schematics. Elsevier (1998).

28. Blanchard, R. J., Blanchard, D. C. Crouching as an index of fear. Journal of Comparative and Physiological Psychology. 67 (3), 370-375 (1969).

29. Choy, K. H. C., Yu, J., Hawkes, D., Mayorov, D. N. Analysis of vigilant scanning behavior in mice using twopoint digital video tracking. Psychopharmacology. 221 (4), 649-657 (2012).

30. Casanova, J. P. et al. A role for the interoceptive insular cortex in the consolidation of learned fear. Behavioral Brain Research. 296, 70-77 (2016).

31. Casanova, J. P., Aguilar-Rivera, M., Rodriguez, M., Coleman, T. P., Torrealba, F. The activity of discrete sets of neurons in the posterior insula correlates with the behavioral expression and extinction of conditioned fear. Journal of Neurophysiology. 120 (4), 1906-1913. (2018).

32. Contreras, M., Ceric, F., Torrealba, F. Inactivation of the interoceptive insula disrupts drug craving and malaise induced by lithium. Science. 318 (3850), 655-658 (2007).
33. McNaughton, N., Corr, P. J. A two-dimensional neuropsychology of defense: fear/anxiety and defensive distance. Neuroscience and Biobehavioral Reviews. 28 (3), 285-305 (2004).

34. Blanchard, D. C., Canteras, N. S., Markham, C. M., Pentkowski, N. S., Blanchard, R. J. Lesions of structures showing FOS expression to cat presentation: effects on responsivity to a cat, cat odor and nonpredator threat. Neuroscience and Biobehavioral Reviews. 29 (8), 1243-1253 (2005).

35. Maswood, S., Barter, J. E., Watkins, L. R., Maier, S. F. Exposure to inescapable but not escapable shock increases extracellular levels of 5-HT in the dorsal raphe nucleus of the rat. Brain Research. 783 (1), 115-120 (1998).

36. Maier, S. F. et al. The role of the amygdala and dorsal raphe nucleus in mediating the behavioral consequences of inescapable shock. Behavioral Neuroscience. 107 (2), 377 (1993).

37. Maier, S. F, Grahn, R. E, Watkins, L. R. 8-OHDPAT microinjected in the region of the dorsal raphe nucleus blocks and reverses the enhancement of fear conditioning and interference with escape produced by exposure to inescapable shock. Behavioral Neuroscience. 109 (3), 404-412 (1995).

38. Hartley, C. A., Gorun, A., Reddan, M. C., Ramirez, F., Phelps, E. A. Stressor controllability modulates fear extinction in humans. Neurobiology of Learning and Memory. 113, 149-156 (2014).

39. Staples, L. G., Hunt, G. E., Cornish, J. L., McGregor, I. S. Neural activation during cat odor-induced conditioned fear and 'trial 2' fear in rats. Neuroscience and Biobehavioral Reviews. 29 (8), 1265-1277 (2005). 
40. Blanchard, D. C. et al. Failure to produce conditioning with low-dose trimethylthiazoline or cat feces as unconditioned stimuli. Behavioral Neuroscience. 117 (2), 360-368 (2003).

41. Rosen, J. B., Asok, A., Chakraborty, T. The smell of fear: innate threat of 2,5-dihydro-2,4,5-trimethylthiazoline, a single molecule component of a predator odor. Frontiers in Neuroscience. 9, 292 (2015).

42. Verbitsky A., Dopfel, D., Zhang, N. Rodent models of post-traumatic stress disorder: behavioral assessment. Translational Psychiatry. 10 (1), 132 (2020).

43. Fendt, M. et al. Predator odor but not TMT induces 22$\mathrm{kHz}$ ultrasonic vocalizations in rats that lead to defensive behaviors in conspecifics upon replay. Scientific Reports. 8, 11041 (2018).

44. Fragale, J. E. et al. Dysfunction in amygdala-prefrontal plasticity and extinction-resistant avoidance: A model for anxiety disorder vulnerability. Experimental Neurology. 275 (Pt 1), .59-68 (2016). 